\title{
Bioanalysis
}

\section{White Paper on recent issues in bioanalysis: rise of hybrid LBA/LCMS immunogenicity assays (Part 2: hybrid LBA/LCMS biotherapeutics, biomarkers \& immunogenicity assays and regulatory agencies' inputs)}

The 2017 11th Workshop on Recent Issues in Bioanalysis (11th WRIB) took place in Los Angeles/Universal City, California on 3-7 April 2017 with participation of close to 750 professionals from pharmaceutical/biopharmaceutical companies, biotechnology companies, contract research organizations and regulatory agencies worldwide. WRIB was once again a 5-day, weeklong event - a full immersion week of bioanalysis, biomarkers and immunogenicity. As usual, it was specifically designed to facilitate sharing, reviewing, discussing and agreeing on approaches to address the most current issues of interest including both small and large molecule analysis involving LCMS, hybrid ligand binding assay (LBA)/LCMS and LBA approaches. This 2017 White Paper encompasses recommendations emerging from the extensive discussions held during the workshop, and is aimed to provide the bioanalytical community with key information and practical solutions on topics and issues addressed, in an effort to enable advances in scientific excellence, improved quality and better regulatory compliance. Due to its length, the 2017 edition of this comprehensive White Paper has been divided into three parts for editorial reasons. This publication (Part 2) covers the recommendations for biotherapeutics, biomarkers and immunogenicity assays using hybrid LBA/LCMS and regulatory agencies' inputs. Part 1 (LCMS for small molecules, peptides and small molecule biomarkers) and Part 3 (LBA: immunogenicity, biomarkers and pharmacokinetic assays) are published in Volume 9 of Bioanalysis, issues 22 and 24 (2017), respectively.

First draft submitted: 21 September 2017; Accepted for publication: 29 September 2017; Published online: 5 December 2017

\section{Index with page numbers}

Introduction

1896

Discussion topics

1898

Discussions, consensus and conclusion

1899

Key input from regulatory agencies

1905

Recommendations

1907

References

1910

The 11th edition of the Workshop on Recent Issues in Bioanalysis (11th WRIB) was held in Los Angeles/Universal City, California on 3-7 April 2017 with attendance of over 750 professionals from pharmaceutical/biopharmaceutical companies, biotechnology companies, contract research organizations and regulatory agencies worldwide. The workshop included three sequential core workshop days, two additional advanced specialized sessions and four training courses that together spanned an entire week to allow exhaustive and thorough coverage of the current major issues in bioanalysis, biomarkers and immunogenicity.

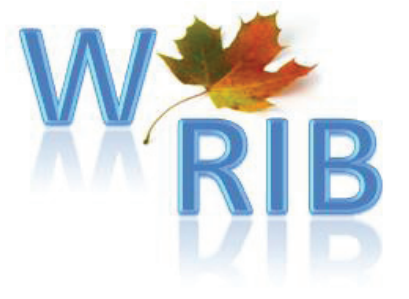

Hendrik Neubert ${ }^{1}$, An Song ${ }^{2}$, Anita Lee ${ }^{3}$, Cong $\mathrm{Wei}^{4}$, Jeff Duggan ${ }^{5}$, Keyang $\mathrm{Xu}^{2}$, Eric Woolf ${ }^{6}$, Chris Evans ${ }^{7}$, Joe Palandra', Omar Laterza ${ }^{3}$, Shashi Amur ${ }^{8}$, Isabella Berger $^{9}$, Mark Bustard ${ }^{10}$, Mark Cancilla ${ }^{6}$, Shang-Chiung $\mathrm{Chen}^{2}$, Seongeun (Julia) $\mathrm{Cho}^{8}$, Eugene Ciccimaro ${ }^{11}$, Isabelle Cludts $^{12}$, Laurent Cocea ${ }^{10}$, Celia D'Arienzo ${ }^{11}$, Lieza Danan-Leon ${ }^{13}$, Lorella Di Donato $^{14}$, Fabio Garofolo*,15, Sam Haidar ${ }^{8}$, Akiko IshiiWatabe $^{16}$, Hao Jiang ${ }^{11}$, John $\mathrm{Kadavil}^{8}$, Sean Kassim ${ }^{8}$, Pekka Kurki ${ }^{17}$, Olivier Le Blaye ${ }^{18}$, Kai Liu $^{19}$, Rod Mathews², Gustavo Mendes Lima Santos ${ }^{20}$, Makoto Niwa ${ }^{21}$, João PedrasVasconcelos ${ }^{8}$, Mark Qian²2, Brian Rago ${ }^{23}$, Ola Saad², Yoshiro Saito ${ }^{16}$, Natasha Savoie $^{24}$, Dian Su², Matthew Szapacs ${ }^{7}$, Nilufer Tampal ${ }^{8}$, Stephen Vinter ${ }^{25}$, Jian Wang ${ }^{11}$, Jan Welink ${ }^{26}$, Emma Whale ${ }^{25}$, Amanda Wilson ${ }^{27}$ \& Y-J Xue ${ }^{28}$

Disclosure: The views expressed in this article are those of the authors and do not reflect official policy of the US FDA, Europe EMA, France ANSM, UK MHRA, Austria AGES, Brazil ANVISA, Health Canada, Japan MHLW and Finland Fimea. No official endorsement by the FDA, EMA, ANSM, Health Canada, MHRA, AGES, ANVISA, MHLW or Fimea is intended or should be inferred.

Author affiliations can be found on the last page.

newlands

press part of 
Abbreviations

\begin{tabular}{|c|c|}
\hline ADA: & Antidrug antibody \\
\hline AE: & Adverse event \\
\hline Anti-miRNA/anti-miR: & Anti-micro RNA \\
\hline BA: & Bioavailability \\
\hline BE: & Bioequivalence \\
\hline BAV: & Biomarker assay validation \\
\hline BMV: & Bioanalytical method validation \\
\hline CDR: & Complementarity-determining region \\
\hline CFR: & Code of Federal Regulations \\
\hline CRO: & Contract Research Organization \\
\hline CSF: & Cerebrospinal fluid \\
\hline EIR: & Establishment inspection report \\
\hline EWG: & Expert working group \\
\hline FFP: & Fit For Purpose \\
\hline FIH: & First in human \\
\hline GLP: & Good Laboratory Practices \\
\hline Hybrid LBA/LCMS: & IA-LCMS \\
\hline HRMS: & High resolution mass spectrometry \\
\hline IA: & Immunoaffinity \\
\hline IND: & Investigational new drug \\
\hline IWG: & Implementation Working Group \\
\hline LBA: & Ligand binding assay \\
\hline LCMS: & Liquid chromatography mass spectrometry \\
\hline LLOQ: & Lower Limit of Quantitation \\
\hline mAb: & Monoclonal antibody \\
\hline pAb: & Polyclonal antibody \\
\hline PC: & Positive Control, used in an immunogenicity assay \\
\hline PD: & Pharmacodynamic \\
\hline PK: & Pharmacokinetic \\
\hline PTM: & Post-translational modifications \\
\hline SDS: & Sodium dodecyl sulfate \\
\hline TPD: & Therapeutic Products Directorate \\
\hline TK: & Toxicokinetic \\
\hline WRIB: & Workshop on Recent Issues in Bioanalysis \\
\hline
\end{tabular}

As in previous years, the 11th WRIB gathered a wide diversity of international industry opinion leaders and regulatory authority representatives working in the field of small and large molecule bioanalysis, to facilitate the sharing and discussions focused on improving quality, increasing regulatory compliance and achieving scientific excellence on bioanalytical issues.

The active contributing chairs included Dr Eric Yang (GlaxoSmithKline), Dr Jan Welink (EMA), Dr An Song (Genentech), Dr Hendrik Neubert (Pfizer), Dr Fabio Garofolo (Angelini Pharma), Dr Shalini Gupta (Amgen), Dr Binodh DeSilva (Bristol-Myers Squibb) and Dr Lakshmi Amaravadi (Sanofi). 


\section{Key terms}

Aptamers: Synthetic oligonucleotides that adopt a 3D structure and bind to target molecules.

Immunoglobulin isotype: Slight phenotypic variations within the immunoglobulin gene family that encode for variant immunoglobulin heavy and light chains.

Oligonucleotide: Short nucleic acid polymers, made up of 13-25 nucleotides.

Isotopomer: Any isomer of an organic compound differing only in the position of an isotope.

The participation of regulatory agency representatives continued to grow at WRIB [1-15], covering topics and discussions in bioanalysis, biomarkers and immunogenicity:

- Dr Sean Kassim (US FDA), Dr Sam Haidar (US FDA), Dr Seongeun (Julia) Cho (US FDA), Dr Nilufer Tampal (US FDA), Dr Jan Welink (EU EMA), Dr Olivier Le Blaye (France ANSM), Mr Stephen Vinter (UK MHRA), Ms Emma Whale (UK MHRA), Dr Isabella Berger (Austria AGES), Mr Gustavo Mendes Lima Santos (Brazil ANVISA) and Dr Mark Bustard (Health Canada) - regulated bioanalysis.

- Dr Shashi Amur (US FDA), Dr John Kadavil (US FDA) and Dr Yoshiro Saito (Japan MHLW-NIHS) - biomarkers.

- Dr Kara Scheibner (US FDA), Dr João Pedras-Vasconcelos (US FDA), Dr Pekka Kurki (Finland Fimea), Dr Isabelle Cludts (UK MHRA-NIBSC), Dr Laurent Cocea (Health Canada) and Dr Akiko Ishii-Watabe (Japan MHLW-NIHS) - immunogenicity.

The 11th WRIB was designed to cover a wide range of topics, in bioanalysis, biomarkers and immunogenicity, suggested by members of the community. Industry experts and regulatory representatives participated in daily working dinners and delivered lectures with each themed session culminating in an open panel discussion between the presenters, regulators and conference attendees in order to finalize the consensus items to be included in this White Paper.

At this year's WRIB, 40 recent issues ('hot' topics) were addressed and distilled into a series of relevant recommendations. Presented here are the exchanges, consensus and resulting recommendations on these 40 topics, which are separated into the following areas:

- LCMS for small molecules:

- Biomarkers and peptide bioanalysis (5 topics);

= Bioanalytical regulatory challenges (7 topics);

- Hybrid ligand binding assay (LBA)/LCMS for biotherapeutics:

= Pharmacokinetic (PK) assays and biotransformations (2 topics);

- Immunogenicity assays (1 topic);

- Biomarker assays (5 topics);

- LBA for biotherapeutics:

= Immunogenicity assays (11 topics);

$=$ PK assays (4 topics);

= Biomarker assays (5 topics).

In addition to the recommendations on the aforementioned topics, there is also an additional section that focuses specifically on several key inputs from regulatory agencies.

Due to its length, the 2017 edition of this comprehensive White Paper has been divided into three parts for editorial reasons. This publication (Part 2) covers the recommendations for biotherapeutics, biomarkers and immunogenicity assays using hybrid LBA/LCMS and regulatory agencies' inputs. Part 1 (LCMS for small mol- 
ecules, peptides and small molecule biomarkers) and Part 3 (LBA: immunogenicity, biomarkers and PK assays) are published in Volume 9 of Bioanalysis, issues 22 and 24 (2017), respectively.

\section{Discussion topics}

The topics detailed below were considered as the most relevant 'hot topics' based on feedback collected from the 10th WRIB attendees. They were reviewed and consolidated by globally recognized opinion leaders before being submitted for discussion during the 11th WRIB. The discussions, consensus and conclusions are in the next section and a summary of the key recommendations is provided in the final section of this manuscript.

\section{PK \& antidrug antibody assays \& biotransformation}

Understanding why \& when to use innovative hybrid LBA/LCMS for immunogenicity assays

Building on the 2015 and 2016 White Paper recommendations and answering remaining questions for applying hybrid LBA/LCMS for immunogenicity assays: LBA uses antidrug antibody (ADA) concentrations or titers, whereas hybrid LBA/LCMS uses ADA concentrations. What are the consequences of using titers versus concentrations? The positive control (PC) antibody is a surrogate of the ADA for establishing assay sensitivity and monitoring assay performance. Can we only use a monoclonal antibody for method development and validation? If a polyclonal antibody is needed, can we monitor animal surrogate peptides to estimate sensitivity? What is the next step in ADA assays by hybrid LBA/LCMS since it was shown in 2015 and 2016 that it is possible to use hybrid LBA/LCMS? Why and when is it an appropriate platform for ADA assays? What do regulators think about ADA by hybrid LBA/LCMS? Are they ready to accept the results of hybrid LBA/LCMS along with LBA? Were there any examples of submissions to regulatory agencies using ADA by hybrid LBA/LCMS? Can we consider mixed applications of LBA and LCMS for immunogenicity assessment, using LBA for screening/confirmatory assays and LBA/LCMS for characterization on the confirmed positive samples (relative concentrations of ADA isotypes) taking advantage of both techniques? The established LBA/LCMS assays are quite generic for different projects. A tiered approach was established based on LBA's features (qualitative assay and prone to interferences from targets or other proteins that cause false positives). Is the tiered approach or relative concentration approach more practical for LBA/LCMS assays? What are the advantages of using the tiered approach if we can already do semiquantitation for ADA? What is the clinical relevance of ADA isotyping by hybrid LBA/LCMS?

Bioanalytical issues for oligonucleotides: siRNA, miRNAs \& modified mRNA: innovative LCMS \& highresolution mass spectrometry options to overcome old bioanalytical problems

Bioanalysis of oligonucleotides often encounters analytes that are analogs of established authentic standards. These analogs (such as metabolites, degradants or impurities) may be pharmacologically active and are important to be included as part of the analysis. In the absence of authentic standards, what are the best practices to quantify and report results? Do the same or different principles apply for MS-based methodology versus hybridization-based methodology? When assaying oligonucleotides, what is measured, how and why (e.g., breakdown products in vivo, need for complete metabolite profiling)? What are the criteria for choosing the right method to answer the required question? Are LCMS and high-resolution mass spectrometry (HRMS) the best platforms to achieve the selectivity needed? What is important to consider for oligonucleotide bioanalytical method validation $(\mathrm{BMV})$ ? What are the current industry standards for applying and supporting a fit-for-purpose (FFP) validation with regulators?

New insights in understanding the biotransformation of biotherapeutics by hybrid LBA/LCMS: what are we actually measuring?

Can we presently consider the high specificity of hybrid LBA/LCMS the current 'gold standard' in regard to what can be or needs to be quantified as a result of metabolism/catabolism (e.g., oxidation, deamidation, depegylation and truncation) versus traditional LBA alone? Why? Use of multiple signature peptides in understanding what is measured: how can different results coming from hybrid LBA/LCMS PK assays help to understand the biotransformation of the biotherapeutic? What are the current approaches to overcome the impact of biotherapeutic deamidation and oxidation on quantification? How can deamidation artifacts be reduced or controlled in hybrid LBA/LCMS assays? How is methionine oxidation evaluated and reversed? Should formulation stability data trigger the development of quantitative hybrid LBA/LCMS assays? Would an in vitro system (microsomes, hepatocytes) better predict potential metabolism or modifications in vivo? How early in the discovery process should in vivo site-specific modifications to therapeutic antibodies be investigated? Prior to Cynomolgus monkey studies 
(e.g., in mice?), should all site-specific modifications be investigated in vivo (e.g., when the modification does not occur in the binding regions of the antibody)? Should measurement of in vivo modifications continue in early development (investigational new drug enabling toxicology studies, first in human) on an exploratory basis if the extent of modification was acceptable in discovery studies?

\section{Biomarker assays}

Assessing drug-target engagement in tissue with new hybrid LBA/LCMS

What is the current industry experience with hybrid LBA/LCMS to measure, in a single assay at the site of action, drug exposure and target engagement? Can (tissue) biomarker measurements be informative without having established accuracy? What are the critical method development steps and best practices that should be taken to ensure assay qualification: protein biomarker recovery from tissue; normalizing protein biomarker tissue measurements (wet weight, total protein, marker proteins); strategies to remove/compensate for blood contamination? Are there any tissue handlers/liquid handlers that have proven particularly helpful for improving throughput, while maintaining protein integrity?

\section{Recent developments in protein biomarker quantification by hybrid LBA/LCMS}

How can small molecule sample preparation techniques be leveraged for use with large molecules: executing and automating protein precipitation for peptide immunoaffinity LCMS? What are the merits of using nanoflow/ nanospray techniques in quantitative protein LCMS/MS? What is the current status of multiplexing in hybrid LBA/LCMS? What are the strategies to deal with 'poor' antiprotein-based capture? What is the current industry experience in using 'bad' LBA antiprotein antibodies in immunoaffinity (IA) for hybrid LBA/LCMS to enrich target protein? How often does it work? When are binding partners and soluble ligand receptors considered as part of assay development?

\section{Protein biomarker proteoforms by hybrid LBA/LCMS}

Many candidate protein biomarkers exist in multiple proteoforms in vivo. Hybrid LBA/LCMS assays have the potential to be highly selective, and therefore recognize specific proteoforms. When is it important to develop proteoform specific biomarker assays? How does one determine which proteoform to measure? What are the advantages of using a biological matrix where only the endogenous protein biomarker was depleted while retaining all other biological components of the matrix?

\section{Using aptamers for hybrid LBA/LCMS assays}

What are the pros and cons for using aptamers more routinely in affinity purification techniques? What are the advantages of aptamers as IA reagents versus traditional antibody approaches?

Progress in the recommendations for the validation of protein biomarkers by hybrid LBA/LCMS in support of clinical programs: challenges \& opportunities

Hybrid LBA/LCMS assays are now routinely used for biomarker measurement, not only in discovery but also in support of clinical studies. What experiments should be conducted during method validation of hybrid LBA/ LCMS methods? Do you agree that hybrid LBA/LCMS FFP biomarker assay validation (BAV) in support of relevant clinical studies and label claims should at least include intra-assay precision, inter-assay precision, lower limit of quantitation, analyte stability, dilution linearity, parallelism and recovery? Can we reach consensus and a recommendation?

\section{Discussions, consensus \& conclusions}

PK \& ADA assays \& biotransformations

Understanding why \& when to use innovative hybrid LBA/LCMS for immunogenicity assays

Hybrid LBA/LCMS assays have unique features such as direct ADA measurement [16,17], good detection, specificity and powerful multiplexing/isotyping capabilities. The capture portion of the assay is independent of bivalent binding and less affected by drug interference. The indirect formats, by capturing the drug-ADA complex, can mitigate drug interference [18]. Previous discussions of the innovative utility of hybrid LBA/LCMS for immunogenicity assays, as discussed in the 2015 and 2016 White Papers [11-14], have generated high interest among industry and regulators to understand the advantages and challenges of using the LCMS platform versus the traditional LBA platform. This year's discussion was built on the 2015 and 2016 White Paper recommendations 
and attempted to address the remaining questions from the 2016 discussions. The possible use of hybrid LBA/ LCMS for ADA assays was demonstrated in 2015 and 2016; hence, this year's discussion focused on the questions 'Why?' and 'When?' to perform these hybrid assays. It was concluded that the advantages of using LCMS include better specificity, reduced drug interference and reduced false positives. Currently, hybrid LBA/LCMS may be the platform of choice when there are unique challenges that cannot easily be overcome by LBA, such as novel or unusual matrices (e.g., mucosal secretions), when LBA assays show poor selectivity (e.g., low drug tolerability) or as a confirmatory tool of ADA positive sample sets.

The tiered approach for immunogenicity assays was established based on LBA's features (qualitative/semiquantitative assays and prone to interferences from targets or other proteins that cause false positives). For hybrid LBA/LCMS, a similar tiered approach can actually be followed when LCMS is used as a detector similar to the detection antibody for LBA. However, the relative concentration approach is more practical and simple for hybrid LBA/LCMS assays, because hybrid assays can specifically measure the relative concentrations of ADA with less false positive and drug interference; therefore, the first two tiers, screening and confirmation, may not be necessary. Regarding ADA data reporting, LBA uses ADA titers, whereas hybrid LBA/LCMS uses ADA titers or relative ADA concentrations, resulting in two types of results. However, both titers and concentrations indicate the strength of the immune response. Sponsors interested in using hybrid LBA/LCMS immunogenicity assays are encouraged to contact regulators to discuss the appropriate approach for determining cut points and reporting ADA levels.

For LBA assays, the PC antibody preparation is a surrogate ADA used for establishing assay sensitivity and monitoring assay performance. Similarly, for hybrid methods, a PC is still needed for method development and validation. A PC for hybrid LBA/LCMS assays may contain the same signature peptides used for the ADAs in human samples to obtain a better estimation of the assay sensitivity. To this end, a humanized PC (or the PC containing human $\mathrm{Fc}$ ) can be used because the signature peptides are located in the highly conserved $\mathrm{Fc}$ region. However, using peptides that are unique to the PC and do not occur in ADA can also be useful to enable the use of nonhumanized PCs where that is needed.

It was discussed whether mixed applications of LBA and hybrid LBA/LCMS for immunogenicity assessment could be considered. That is, LBA could be used for screening/confirmatory assays and hybrid LBA/LCMS for characterization using the confirmed positive sample to determine the relative concentrations of ADA isotypes. This combination approach allows for the application of advantages from both techniques. This approach was considered more favorable than using hybrid LBA/LCMS alone for all assays. Initial testing with an LBA would allow regulators to review results obtained using a traditional approach for screening positives while the hybrid LBA/LCMS could be used for specificity testing and additional characterization. In addition, in-parallel comparison of LBA and hybrid assay results (titers vs relative concentrations of ADA) from a same study would permit to evaluate the correlation of two methods in ADA assessment. As hybrid LBA/LCMS methodologies gain in use, differences in drug tolerance and sensitivity of the LBA versus hybrid LBA/LCMS would need to be considered when interpreting the screening, confirmatory and characterization results in aggregate and ultimately the immunogenicity impact on efficacy/safety. The clinical relevance of ADA isotyping by hybrid LBA/LCMS was discussed. At this time, ADA isotyping is not a default requirement by regulators. Currently, isotyping analysis is primarily driven by clinical safety signals, and is conducted case by case. When needed, specificity, multiplexing capability and capacity of hybrid assays would greatly benefit ADA isotyping and characterization.

Hybrid LBA/LCMS immunogenicity assays are currently easier to implement in discovery stage or in early exploratory clinical investigations. Industry is encouraged to generate more exploratory data and to seek regulatory agency feedback prior to applying the approach in later stage clinical studies. Current regulatory feedback indicates that US FDA and Japan MHLW have yet to see submissions for clinical immunogenicity assessment using hybrid LBA/LCMS. The FDA would like to have sponsors to present seminars to regulators on how these types of methods could be validated and to see the comparison between titer results (LBA) and concentration results (LCMS) to get some confidence around the correlation. They see potential for these methods in a regulated environment, but would like to open a dialog to get specifics on their application, in particular regarding assay validation.

Bioanalytical issues for oligonucleotides: siRNA, miRNAs \& modified mRNA: innovative LCMS \& HRMS options to overcome old bioanalytical problems

An anti-miRNA is a single-stranded synthetic oligonucleotide that sequesters, based on sequence specificity, a target miRNA in diseased cells to form an inactive heteroduplex. Anti-miRNAs of different designs targeting 
various miRNAs are currently being developed as therapeutics for various disease indications. Bioanalysis is an integral part of the discovery and development of anti-miRNA therapeutics, providing crucial information on nonclinical PK/toxicokinetic (TK), clinical PK and for PK/pharmacodynamic (PD) correlations.

When assaying oligonucleotides, one needs to understand how critical the breakdown products are with regard to safety and efficacy of the products. It is a case-by-case issue; however, understanding what breakdown products are considered critical for project interpretation will assist the bioanalytical scientist in designing a suitable analytical strategy. LCMS and HRMS are considered the preferred platforms for a bioanalytical measurement of well-defined molecular moieties. However, in some situations, hybridization-based methods could complement LCMS and HRMS methods to allow simultaneous bioanalytical measurement of different molecular moieties with the same sequence and having the same hybridization potential with target of interest. Appropriate storage of study samples is also a point of focus to avoid stability issues impacting future additional investigations. When validating an oligonucleotide assay, all standard BMV parameters apply.

Bioanalysis of oligonucleotides often encounters analytes that are analogs of established authentic standards. These analogs (such as metabolites, degradants or impurities) may be pharmacologically active and are important to be included as part of the analysis. It is uncommon to have access to authentic standards for all of the possible analogs, and therefore in the absence of authentic standards, the assay is relative quantitative to a surrogate standard. The utility of either MS or hybridization methodologies for analog analysis incur a number of assumptions. Whenever possible, it is important to try to have analog analytical standards with sequences that will bracket what the assay is trying to capture; however, data interpretation still requires drawing on a number of assumptions.

New insights in understanding the biotransformation of biotherapeutics by hybrid LBA/LCMS: what are we actually measuring?

During drug discovery, therapeutic antibodies under consideration are first evaluated for chemical stability in formulation buffer, undergoing stressed conditions including extremes of heat and $\mathrm{pH}$. Although the nature of these modifications is not enzymatic, they may be indicative of sites of in vivo metabolism/catabolism when dosed, which can potentially impact pharmacological and toxicological properties. Qualitative in vitro stability methods in biological matrices (e.g., plasma/blood stability) or in vivo could be used before quantitative methods are developed for this determination.

Isomerization of aspartic acid and deamidation of asparagine are two common amino acid modifications that are of particular concern, especially if they occur within the complementarity-determining region of a therapeutic antibody since binding affinity and potency can be affected [19]. The 'stressed' compound undergoes full characterization by intact mass LCMS as well as peptide mapping. The information from these studies can be combined to reveal site-specific modifications to the protein. With this information, bioanalytical assays that quantify the extent of in vivo metabolism/catabolism of the molecule in either preclinical or clinical studies can be developed and implemented. In order to comprehensively investigate biotransformation of biotherapeutics, ideally the whole protein should be monitored. However, due to technology limitations, subdomain monitoring and LCMS analysis of multiple signature peptides at different segments of the protein could be used to understand biotransformations at different regions of the protein. These data can then be pieced together to provide a complete picture of the biotransformation of the protein.

The timing of the investigation of in vivo site-specific modifications to therapeutic antibodies in the discovery process depends on the knowledge base of the type of molecules. If there is a history of a similar class of molecules having known issues, then it is recommended to start the investigation early in discovery. Also, if it is a novel molecule and the risk is considered high, regulatory scrutiny would likely be increased, presenting the need for early investigations. The sites that require investigation, for example, when the modification does not occur in the binding regions of the antibody, also depend on the knowledge of the molecule and the potential impact on PK, efficacy or safety. The extent of the investigation and whether exploratory measurement of in vivo modifications should continue in early development (investigational new drug enabling toxicology studies, first in human) may depend on what degree of modification is considered acceptable by the sponsor. The sponsor will need to justify what they are monitoring, and provide appropriate data to substantiate the scientific rationale.

Traditional LBA methods have long been the gold standard for biotherapeutic assays used to understand metabolism/catabolism. With the increased use and benefits of hybrid LBA/LCMS, it was discussed whether this technology has supplanted LBA as the gold standard. It was concluded that hybrid LBA/LCMS can be very useful in understanding metabolism/catabolism; however, it still depends on how the affinity capture method is designed. When affinity capture reagents are more generic and focused on enrichment, the downstream specific- 
ity of the LCMS can be used confidently to identify and later quantify these catabolic products. However, with more specific capture reagents, if they are sensitive to the biotransformations in question, then similar limitations and biases as LBA could be faced with hybrid LBA/LCMS using these same reagents.

It is essential to understand whether the biotherapeutic deamidation and oxidation can impact quantification. It is important to reduce or control deamidation artifacts and evaluate or reverse methionine oxidation during sample preparation. Traditional LCMS proteomic approaches for reducing deamidation artifacts can be applied to hybrid methods (e.g., reducing the digestion time as much as possible, adding organic solvents). When possible, methionine oxidation should be avoided altogether by selecting a different signature peptide for quantification.

\section{Biomarker assays}

Assessing drug-target engagement in tissue with new hybrid LBA/LCMS

Accessibility and innovative integration of advanced technologies have accelerated the development of hybrid LBA/LCMS, which has become an important bioanalytical platform to verify novel targets in discovery and confirm promising targets and biomarkers in early clinical development. Bioanalytical methods based on IA-LCMS to quantitatively assess drug-target engagement in serum and tissue biopsies have been used to support target selection, preclinical candidate selection, dose projection and in early clinical studies for prompt proof of concept.

One bioanalytical strategy is to design, develop and verify hybrid LBA/LCMS quantitative workflows with sufficient rigor for application in decisional studies. These hybrid methods that support discovery program progression can be refined and qualified during subsequent evaluation in different animal species and tested on human samples, which can be purchased via commercial vendors or ideally through established collaborations with academic institutions or medical centers. Establishing a comprehensive workflow strategy that confirms drug binding to target in the specified tissue at levels considered efficacious was the focus of this discussion. Execution of this workflow in exploratory/investigational clinical studies and comparing validated/qualified measurement of circulating drug levels collected at the same time as tissue biopsies with total drug levels in tissue can provide key information for future development.

Hybrid LBA/LCMS offers the potential to fill a current gap in analytical techniques by directly measuring drug exposure and target engagement, two of the three critical pharmacological questions, with a single assay [20]. Unlike LBA, this approach is less dependent on reagent specificity and therefore less subject to interference. Unlike flow cytometry, hybrid LBA/LCMS does not rely on intact viable cells and it allows for accurate quantification in frozen tissue, which could be challenging with other platforms. Current industry experience with hybrid LBA/LCMS assays used to measure drug exposure and target engagement in a single assay at the site of action in tissues is limited but quickly emerging. When sample availability for a duplex assay is limited, it is recommended to perform an FFP validation for both end points. Biomarker measurements (in tissue) can be informative without having established accuracy. If such a study is submitted to an agency, indicating the limitations of the assay may be sufficient for the agency (transparency and communication are key). A validation workplan where PK is the primary end point (which requires a fully validated method), and target engagement is the exploratory/secondary end point (where an FFP method is sufficient) should be considered.

Consensus was reached on the method development steps and best practices that should be taken to ensure tissue assay qualification. After homogenization and extraction, the sample may still have debris remaining, which can be re-extracted with fresh lysis buffer and probed to determine if additional analyte remains. If so, repeat extraction may be warranted. It should be noted that there is currently not much advancement in improving throughput of tissue homogenization. Regarding pellet digestion/antipeptide antibody, in other words, for membrane and structural proteins not quantifiable by other techniques, a very harsh extraction (e.g., with sodium dodecyl sulfate) is an analytical option to fully extract the target. Normalization using wet tissue weight, total protein or a housekeeping protein can be considered; however, these can be difficult to assess (need to confirm they are not changing with the disease). Finally, blood contamination of tissue is sample and tissue-type dependent (i.e., gut mucosal tissue may not have as much blood contamination as a punch biopsy). Instead, bloodderived proteins could be considered to assess blood contamination in the tissue sample.

\section{Recent developments in protein biomarker quantification by hybrid LBA/LCMS}

Protein immunoaffinity techniques linked to MS (hybrid LBA/LCMS) have solidified their impact in translational research and in clinical analysis $[8,11,14]$. This technique has been on an incredible journey in recent years that enabled growing adaptation through its use by an increasing number of practitioners and experts due to improved assay sensitivity and throughput, new reagents for capture approaches and automation of key steps, 
to name a few factors. Alongside this technological advancement, the application space of hybrid LBA/LCMS proliferated to provide new demonstrated capabilities thereby unlocking new bioanalytical opportunities.

Bioanalysts have advanced the ability from measuring soluble proteins to target engagement, moved from plasma to tissues including small biopsies, from soluble proteins to structural and membrane bound proteins and from concentration analysis to measuring protein synthesis rates. In addition, the progression of biotherapeutic modalities across discovery and development stage gates increasingly requires the use of PK/PD modeling. This has subsequently raised the demand for quantitative measurements of target biomarkers in soluble matrices and tissues. These measurements can be critical as they can inform target validation, occupancy and dose projections and precision medicine approaches for patient stratification. This includes target measurements in normal versus disease matrices; however, obtaining accurate and robust measurements can be challenging. These challenges can come from many factors, such as binding partners, soluble or shed receptors, splice variants, post-translational modifications, just to name a few [21,22].

While comparing current industry standards versus previous White Paper recommendations $[8,11,14]$, there was consensus that one of the methodologies that are proving to be invaluable for tissue-based measurement is extraction of proteins using a protein precipitation step with high organic solvent, pellet digestion followed by the use of antipeptide antibodies for enrichment of tryptic peptides and subsequent measurement by LCMS [23-25]. Even though custom reagent generation is required, this technique enables the use of harsh extraction conditions including strong detergents for maximized recovery of proteins that may otherwise be insoluble, for example, membrane/structural proteins. The approach can be multiplexed and is sensitive. No antiprotein antibody is needed, thereby bypassing the capture efficiency challenge of antiprotein antibodies.

Hybrid LBA/LCMS can be extended from concentration analysis to the determination of synthesis rates of therapeutic targets using pulse chase studies in human subjects with stable isotope-labeled amino acids [26]. The half life of target proteins is frequently a sensitive parameter in mechanistic PK/PD modeling of biotherapeutics. The accurate measurement of physiologically relevant protein turnover in humans is needed to reduce the uncertainty in PK/PD model-based predictions of the therapeutic dosing regimen, for example, in first-inhuman clinical trials. Serial immunoaffinity enrichment of proteins from serum and subsequently from remaining supernatants prior to digestion and LCMS has been confirmed to provide sensitive and reproducible turnover data irrespective of the order of analysis. The sample preparation, which involves serial immunoaffinity extraction of sample supernatant, does not affect isotopomer ratios. This extends the use of valuable clinical pulse chase sample sets as subsequent protein turnover analysis from the supernatants is possible as isotopomer ratios for other proteins remain unchanged by previous sample manipulation.

The use of hybrid LBA/LCMS with nanoflow/nanospray is now more established in the analysis of protein biomarkers. In fact, it offers a unique way to meet these biological challenges associated with protein tissue measurements. Nanoflow, which uses sub- $\mu \mathrm{l} / \mathrm{min}$ flow rates, is used routinely for discovery but is also being used more often in support of clinical programs. It is mostly employed when sample is limited for which the benefits are reduced ionization suppression and enhanced sensitivity. However, limitations of throughput and robustness still need to be overcome on some platforms. The use of automation in this field is increasing for digestions, immunoaffinity and protein precipitation-based approaches. Some best practices can be learned and adapted from the small molecule LCMS community. However, for precipitation-based workflows, it should be considered that since not all proteins precipitate, conditions need to be optimized, for example, solvents, temperature and salts. Multiplexing in hybrid LBA/LCMS is commonly used to measure multiple peptides from the same protein to verify and understand its biology and various proteoforms [27].

Current industry experience using LBA antiprotein antibodies with poor selectivity or specificity in IA for hybrid LBA/LCMS to enrich target protein is limited. For hybrid LBA/LCMS, the antibody criteria are different compared with LBA, which will require more binding specificity. For low affinity antibodies, consider flow-based antiprotein capture rather than bead-based methods to overcome poor antibody affinity. For antiprotein capture reagents, it is important to understand if it competes with the binding partners. Thus, at a biological level, the 'target environment' needs to be understood to develop a meaningful target biomarker assay. An antipeptide antibody approach will work around this issue as well as the issue of interference of soluble ligands, and as a result this approach is being increasingly used.

\section{Protein biomarker proteoforms by hybrid LBA/LCMS}

Many candidate protein biomarkers exist in multiple proteoforms in vivo. Hybrid LBA/LCMS assays have the potential to be very selective, and therefore recognize specific proteoforms. Translational and post-translational 
events can contribute to the generation of proteoforms, and as these proteoforms may have quite different biological activities, the ability to selectively quantify specific proteoforms can be quite important. Alternatively, in some cases, it may be desirable to measure all proteoforms or 'total' protein. It is important to understand the intended use of the biomarker, and the potential impact of proteoforms on that use before assay development. For example, for a predictive biomarker, it might be important to measure only the biologically active species. There is the potential to unintentionally dilute the signal for the purpose of looking at all proteoforms when actually only one is important. For an occupancy assay, all proteoforms that are recognized by the drug candidate should be measured. If a total protein assay is desired, surrogate peptide selection is critical, the 'total' protein assay may be misleading if the peptide is modified and the modified forms not measured. The number and type of proteoforms may not be known, so it is important to clearly define early on what the assay is measuring.

The advantages of using a surrogate matrix versus a biological matrix where only the endogenous protein biomarker was depleted while retaining all other biological components were discussed. It was concluded that either can be used as long as the surrogate matrix is proven parallel in performance with the biological matrix to allow for detectable concentration changes. On occasion, these assays may struggle with nonspecific adsorption, so depleted matrix can better correct for that. If a labeled protein (not peptide) IS is available, it can potentially correct for most analytical issues. However, there are caveats such as membrane proteins, IA efficiency differences, digestion efficiency differences, etc.

\section{Using aptamers for hybrid LBA/LCMS assays}

IA is a crucial component of hybrid LBA/LCMS for the quantitation of low abundance proteins and peptides in biological matrices such as plasma, serum and cerebrospinal fluid. This enrichment technique represents a breakthrough technology in the sense that it allows the quantitation of proteins and peptides at low, previously unattainable, levels. Increased sensitivity and reduced background are distinct advantages when compared with traditional sample preparation methods.

It should be noted that antibodies used in IA may not always be available and may require a long time to generate, or they may be of low quality, poor specificity and/or sensitivity, high cost or show lot-to-lot variability. Thus, alternative affinity purification techniques such as the use of aptamers have been explored.

A case study was discussed, comparing the performance of a novel approach named aptamer affinity (AA)LCMS to the traditional antibody-based approach (IA-LCMS) [28]. It was agreed that one of the major benefits of aptamers is that all reagents are synthetic, resulting in less lot-to-lot variability and no loss of antibody producing cell line issues. The experiment as proof-of-concept worked, offering a potential alternative to antibodies for affinity-enriched methods. Other considerations must be taken into account, however, including, for example, reagent costs, availability and regenerating columns after use. Costs are comparable to antibodies, but depending on the analyte, a mixture of aptamers per reaction may be needed for sufficient capture. Additionally, only a few companies produce aptamers. It is expected to have further discussions and more in-depth recommendations on this technique in the future since aptamers appear to be promising capture reagents for hybrid LBA/LCMS but more case studies are needed for full evaluation.

Progress in the recommendations for the validation of protein biomarkers by hybrid LBA/LCMS in support of clinical programs: challenges \& opportunities

Hybrid LBA/LCMS assays for peptide and protein biomarker quantitation has become a first choice method in a growing number of bioanalytical laboratories. Hybrid assays are moving from drug discovery applications toward supporting later stage clinical programs as these methods are rugged and robust, being validated to current international regulatory guidelines [29-31]. However, an evaluation and understanding of both the biology and variability of the biomarker analyte of interest in the specified matrix of interest are imperative for the development and validation of a suitable hybrid LBA/LCMS assay. Due to the relative ease of obtaining normal or disease state $\mathrm{blood} / \mathrm{sera}$, this is an imperative step in the validation process.

BMV for hybrid LBA/LCMS assays has been discussed multiple times in the past and recommendations were provided $[8,11,14,32]$. However, to date, there is no regulatory guidance/guideline regarding experiments that should be conducted during method validation for hybrid LBA/LCMS methods. BAV was discussed in the 2016 White Paper [15] and in the 2017 document, presently in press [33]. While waiting for official regulatory input, from an industry point of view, it was agreed that a validation plan was needed that listed suitable experiments in order to achieve confidence in the assay, based on the FFP of the assay (i.e., primary or exploratory/secondary end point will drive the decision for FFP or full validation). 
In general, hybrid LBA/LCMS BAV during discovery and early exploratory work follows the same rules as LBA FFP assay validation. Therefore, hybrid LBA/LCMS screening assays only require limited validation (precision, limited accuracy, specificity). For later preclinical work and early clinical proof-of-concept studies with a limited number of samples still used for screening, an FFP validation model is needed. Once biomarker assays are used for later phase studies as a surrogate end point, full validation would be necessary with any limitations well documented [34]. An understanding of the bias/risk that can occur during the use of hybrid LBA/LCMS assay is needed and should be addressed during validation.

As far as BAV is concerned, it should be mentioned that the process of drafting a guidance document is in progress. A public workshop (FDA/Critical Path/industry) was scheduled in June 2017 to obtain feedback on a white paper for evidentiary standards for biomarker qualification for both LBA and LCMS methods (including hybrid LBA/LCMS assays) [35].

\section{Key input from regulatory agencies}

The 11th WRIB continued with its long standing contributions from several international regulatory agencies, including the US FDA, EU EMA, Health Canada, UK MHRA, Austria AGES, France ANSM, Brazil ANVISA, Japan MHLW and Finland Fimea. These agencies shared their views on multiple topics of interest for the global bioanalytical community attending this event, to provide clarification on unresolved issues or expectations. Regulators provided input on a variety of topics across all three core days, including:

- Inspection program updates and observations;

- ICH M10 draft technical document: BMV;

- ICH S3A guideline draft Q\&A: microsampling;

- Unstable analytes;

- Immunogenicity assessment;

- Biomarkers assay inspections and biomarker qualification program (BQP).

Inspection program updates were provided by the US FDA and ANVISA. Drug evaluation program updates were provided by Health Canada.

The US FDA outlined the mission and vision of the Office of Study Integrity and Surveillance (OSIS) following the Office reorganization in early 2015. Bioresearch monitoring (BIMO) at the US FDA has a long tradition of supporting the protection of human subjects by ensuring that clinical and nonclinical research conducted for FDA-regulated products are performed according to the regulations, and adequate to support regulatory decisions. In 2015, the GLP (nonclinical) and Bioavailability/Bioequivalence (BA/BE) Inspection Programs in the FDA's Center for Drug Evaluation and Research (CDER) separated from the existing CDER BIMO inspection programs in the Office of Scientific Investigations to be under OSIS. The new OSIS is now housed in CDER's Office of Translational Sciences. Its vision is to be a world leader in conducting and directing BA/BE and GLP (nonclinical) inspections and, through these inspections, to ensure data submitted to the FDA is adequate to support regulatory decisions. Among OSIS' responsibilities, the Office assigns and conducts inspections, writes establishment inspection reports, makes recommendations and surveillance evaluations of sites and studies, investigates allegations and complaints and collaborates on regulatory actions and enforcement with the Office of Scientific Investigations. In 2016, OSIS directed and conducted 91 analytical BA/BE inspections and 291 clinical BA/BE sites with FDA's Office of Regulatory Affairs (ORA), at sites across the globe. Additionally, OSIS and ORA conducted 28 GLP inspections, including 5 inspections outside the USA. Of note, the BA/BE inspections were more process based in nature than strictly study focused. In fact, the evolving BA/BE inspection approach typically involves audits of parts from several studies instead of all aspects of fewer studies, to gain a better overall assessment of a firm's performance. Some critical aspects that may be inspected include test article accountability, facilities and equipment, blinding codes, randomization schedules, audit trails and prestudy method validation. If concerns are identified, OSIS evaluates the extent of the issues to determine whether they are limited to a specific study or a systemic issue.

Brazil ANVISA also gave an update on their inspection program. Recent ANVISA findings included problems with diluted quality control samples, issues regarding traceability of information and problems with quality assurance activities. 
Health Canada informed that two new Notices have been released by the agency [36,37]. A new Plain Language Labeling Initiative was begun by a new Labeling Division in the Therapeutic Products Directorate, created as of August 2016. The goal of the initiative is to reduce preventable drug harms and support the safe and effective use of drugs by making improvements to ensure health product labels are clear, accurate and easily understandable and to minimize opportunities for confusion with labels, packages or names. The regulator also provided an update to their policy regarding the Notice on stability evaluations [38,39] discussed at last year's forum [14]. It was clarified that the Notice is applicable to bioanalytical methods, primarily for LCMS assays for the measurement of small molecules in comparative bioavailability studies that are pivotal to the drug regulatory assessment of human therapeutic drug products. In order to manage drug submissions without the required stability information in a fair manner, the current process is that a Screening Deficiency Notice is issued. Previously, a Notice of Deficiency was issued immediately. The regulators' expectations for stability data to be submitted have not changed, and for the most part, industry has complied with the Notice. Refer to Part 1 of the 2017 White Paper for further details [40].

Japan MHLW provided an update on the new ICH M10 'BMV'. It was adopted at the ICH Lisbon meeting in June 2016. In July 2016, the M10 informal working group was organized and the final concept paper and business plan were developed [41,42] and released in October 2016. The M10 Expert Working Group (EWG) was then established and the first face-to-face meeting was held in Osaka, Japan in November 2016. The M10 EWG consists of 14 organizations including 7 regulatory members (EMA, FDA, MHLW/PMDA, Health Canada, Swissmedic, ANVISA, MFDS), 5 industry members (EFPIA, JPMA, PhRMA, IGBA, BIO) and 2 observers (WHO, TFDA). The mission of M10 is to provide recommendations on BMV and study sample analyses to obtain the reliable drug/metabolite concentration data in biological matrices, which will be used for regulatory submission. Establishment of the M10 guideline will result in the harmonization of current regional guidelines/ guidances and support streamlined global drug development. The draft guideline is planned for 2018 and the harmonized guideline in 2019. At the ICH Osaka meeting, EWG members performed a gap analysis on controversial issues in current regional guidelines and also discussed the outline of the M10 draft technical document regarding scope, method validation, analytical run and other important issues. Currently, the first draft technical document is in preparation by EWG members, and it will be discussed at the next ICH Montreal meeting in May 2017. The timeline of EWG activities is disclosed in the Work Plan [43].

Japan MHLW provided an update on the ICH S3A guideline draft Q\&A addressing microsampling, previously discussed in the 2016 White Paper [14]. At that time, the draft Q\&A was released for consultation. Public consultation was performed in the USA, EU, Canada and Japan and Implementation Working Group members started to discuss the points raised in the received comments. After considering the comments, the Implementation Working Group will finalize the draft Q\&A in 2017.

US FDA discussed the subject of unstable analytes. FDA regulations (21 Code of Federal Regulations Part 320.29(a)) require that analytical methods used in an in vivo BA or BE study demonstrate the measurement of the actual concentration of the active drug ingredient or therapeutic moiety, or its active metabolite(s), achieved in the body. Instability of an analyte during bioanalysis is a concern as it may result in inaccurate estimation of the analyte concentration, if adequate precautions are not undertaken. Common causes of instability of the parent drug are hydrolysis or degradation, while causes of metabolite instability include hydrolysis of conjugates, hydrolysis followed by transformation and in-source fragmentation. In order to assess instability, knowledge of the chemical structure and major metabolites, which may be found in the drug labeling or in published literature, is paramount. Otherwise, extensive stability studies are needed. The clinical implication of such inaccuracies can be serious. Therefore, study protocols at the clinical site should include appropriate measures to limit the ex vivo conversion of the analytes in the samples from the study subjects (e.g., addition of antioxidants, enzyme inhibitors). Similarly, at the analytical site, attention should focus on controlling for factors that may contribute to the analyte instability (e.g., MS/MS ion source conditions, processing $\mathrm{pH}$ and temperature). A retrospective review of submissions for generic drug applications between 2007 and 2014 found that for analytes with known instability issues, approximately $32 \%$ of the BE studies did not include data to support how the applicants addressed the anticipated instability of the analyte(s). In a few instances, the applicant either repeated the BE study or voluntarily withdrew the application. Therefore, analyte instability should be addressed during method development and validation, as the risk of undetected stability may be difficult to reverse.

Health Canada addressed the topic of unwanted immunogenicity. Assessing the potential impact of drug immunogenicity on the benefit/risk profile of biologics remains a challenge for both drug developers and regulatory agencies. Due to variability in the current ADA detection technologies, and due to inconsistent terminology, clinical signs and symptoms associated with immune-mediated reactions and adverse events could be considered 
in assessing immunogenicity in the absence of detectable ADA. Immunogenicity and allergenicity events are identified in clinical study reports using MedDRA terms such as anaphylactic/anaphylactoid reaction, angioedema, severe cutaneous adverse reactions, immunology and allergy investigations, serum sickness or serum sickness-like reaction. Additional considerations apply to monoclonal antibody therapies. The Biologics and Genetic Therapies Directorate of Health Canada are looking at methods that will allow clinical reviewers to find potential correlations between individual immunogenicity-related adverse events and the benefit/risk profile of biologics. Such correlations could be apparent in specific patient subgroups in clinical trials. Health Canada continues to collaborate with other regulatory agencies to develop a consistent approach to this matter.

US FDA discussed the need for biomarker assay inspections and its Biomarker Qualification Program (BQP). The supporting role of biomarker determinations continues to grow in pivotal PK and PD studies for drug applications. The increased use of biomarker assays has prompted OSIS to broaden its scope of bioanalytical inspections in order to evaluate data and methods associated with biomarker determination in pivotal studies. During an inspection of biomarker measurements, OSIS may take into account special considerations when evaluating parameters such as sensitivity, stability, the calibration curve, reproducibility and specificity/selectivity. These considerations may include the technology used, the biological nature of the analyte, the use of the assay and the objective of the biomarker determination. Therefore, although methods for biomarker determination may present additional challenges compared with traditional PK assays, variables that are evaluated during inspections of PK studies may still apply to biomarker measurements for prestudy validation and in-study analysis. Biomarkers are used in clinical trials for a variety of purposes, ranging from patient stratification to use as primary clinical outcomes. The FDA BQP offers a pathway to encourage development and qualification of biomarkers that can be employed in multiple drug development programs without re-review of biomarker data for the qualified context of use. To date, 6 biomarker qualification submissions to US FDA have been successful and a total of 13 biomarkers have been qualified. To foster biomarker development, FDA has launched two efforts: the Letter of Support is a mechanism to encourage the use of promising biomarkers in exploratory clinical studies to generate additional data to support biomarker development. To date, 13 Letter of Supports have been issued; The Critical Path Innovation Meetings (CPIM) facilitates nonbinding scientific discussions with FDA on novel methods, drug development tools and technologies that can potentially aid drug development. To date, 41 CPIMs have been held. Efforts are also currently underway among various stakeholders to develop evidentiary criteria to support biomarker qualification and to understand the scientific and regulatory considerations needed for the validation of assays used in the qualification efforts. Although, as mentioned above, validation parameters are similar between methods used for PK studies and those needed for biomarker assays. Discussions have been progressing in the bioanalytical community underlining the fact that existing requirements in BMV guidelines [29-31] cannot be applied to biomarker assays as they are, partly because molecular biomarkers endogenously exist in biological fluids.

Japan MHLW informed attendees that to understand the current situation on bioanalysis of biomarkers in Japan, a research group funded by the Japan Agency for Medical Research and Development is planning to survey Japanese pharmaceutical companies on their validation parameters for biomarker measurements. Results are anticipated for the 12th WRIB. A strong partnership of pharmaceutical/biotechnology industries, academia, consortia, patient groups and government will be essential to support harmonization of biomarker method validation.

\section{Recommendations}

Below is a summary of the recommendations made during the 11th WRIB.

\section{PK \& ADA assays \& biotransformations}

- Hybrid LBA/LCMS immunogenicity assays

- Why? - hybrid LBA/LCMS immunogenicity assays if compared with traditional LBA alone may have better specificity (LBA + LCMS), reduced drug interference and reduced false positives.

- When? - Hybrid LBA/LCMS for ADA assays should be used when there are unique challenges that cannot easily be overcome with LBA alone, such as novel or unusual matrices (e.g., mucosal secretions) or when encountering selectivity issues in LBA.

- Sponsors interested in using hybrid LBA/LCMS for ADA assays are encouraged to contact regulators in advance to discuss the appropriate approach to take, particularly regarding assay validation. 
- When deciding to use traditional LBA or innovative hybrid LBA/LCMS for immunogenicity assays, it should be carefully considered as LBA uses titers whereas hybrid LBA/LCMS uses concentrations as data output. Both titers and concentrations indicate the strength of immune response.

- Due to the infancy of hybrid LBA/LCMS for immunogenicity assessment, exploring a mixed application of LBA and hybrid LBA/LCMS could be considered: LBA for ADA screening/confirmatory and hybrid LBA/LCMS for characterization on the confirmed positive samples (relative concentrations of ADA isotypes).

- Bioanalysis of oligonucleotides

= Bioanalysis of oligonucleotides often encounters analytes that are analogs of established authentic standards. These assays provide relative quantitative information.

= LCMS and HRMS are considered the preferred platforms for a bioanalytical measurement of well-defined molecular moieties.

= In some situations, hybridization-based methods could complement LCMS and HRMS methods to allow simultaneous bioanalytical measurement of different molecular moieties with the same sequence and having the same hybridization potential with target of interest.

- When validating an oligonucleotide assay, all standard BMV parameters apply.

- Biotransformation of biotherapeutics

- Hybrid LBA/LCMS is now the gold standard for studying the biotransformation of biotherapeutics.

- Traditional LCMS proteomics approaches for reducing deamidation artifacts are still applicable to hybrid methods (e.g., reducing the digestion time as much as you can, adding organic solvents). Methionine oxidation can be avoided altogether by selecting a different signature peptide.

- Subdomain monitoring and multiple signature peptides at different areas of the protein should be used to understand biotransformation at different regions of the protein. These data can then be pieced together to provide a complete picture of the biotransformation of the protein.

= Formulation stability data may be indicative of sites of in vivo metabolism/catabolism when dosed, which can impact pharmacological and toxicological properties.

- The timing of the investigation of in vivo site-specific modifications to therapeutic antibodies in the discovery process depends on the knowledge base of the type of molecules. If there is a history of a similar class of molecules having safety issues or if it is a novel molecule, then it is recommended to start the investigation early in discovery.

= The sites that require investigation and the extent of the investigation depend on the knowledge of the molecule and the potential impact on PK, efficacy or safety. The sponsor will need to justify what they are monitoring, and to provide the data to substantiate the scientific rationale.

\section{Biomarker assays}

- Target engagement

- Hybrid LBA/LCMS can directly measure drug exposure and target engagement, two critical pharmacological questions, with a single assay.

= When sample availability for a duplex assay is limited, perform an FFP validation for both end points. When submitting to an agency, indicate the limitations of the assay. A validation workplan where PK is the primary end point (which requires a fully validated method) and target engagement is the exploratory/secondary end point (an FFP method is sufficient) could be considered. 
- Best practices to ensure tissue assay development:

- After homogenization and extraction, re-extract with fresh lysis buffer and probe to determine if additional analyte remains. If so, a repeat extraction may be warranted.

- Regarding pellet digestion/antipeptide antibody, that is, for membrane and structural proteins not quantifiable by other techniques, a very harsh extraction (e.g., with sodium dodecyl sulfate) is necessary to efficiently extract the target.

- Normalization using wet tissue weight, total protein or a housekeeping protein can be considered but can also be difficult to assess (need to confirm they are not changing with the disease).

- Blood contamination in the tissue is tissue dependent. Instead, consider blood-derived proteins.

- Recent developments in protein biomarker quantification by hybrid LBA/LCMS

- A methodology that is proving to be invaluable for tissue-based measurement is extraction of proteins using a protein precipitation step with high organic solvent, pellet digestion followed by the use of antipeptide antibodies for enrichment of tryptic peptides and subsequent measurement by LCMS.

- Hybrid LBA/LCMS using nanoflow/nanospray offers a unique way to meet biological challenges associated with tissue protein measurements. It should be employed when improvements to sensitivity are needed or sample volume is low.

- Multiplexing in hybrid LBA/LCMS can be used to measure multiple peptides from the same protein to verify and understand its biology and various proteoforms.

- Advantages of using antipeptide IA enrichment for the analysis of tissue biomarkers are: no antiprotein antibody is needed; workflow suitable for membrane/structural proteins; the harsh denaturing tissue extraction is compatible with the downstream digestion.

- Protein turnover analysis benefits from using hybrid LBA/LCMS in clinical pulse chase studies because the sample preparation does not affect isotopomer ratios.

- For antiprotein capture reagents, it is crucial to understand if it competes with the binding partners. Hence, 'target environment' should be measured to develop a meaningful target biomarker assay. An antipeptide antibody approach will work around this issue as well as the issue of interference of soluble ligands, and as a result this approach is being increasingly used.

- Protein biomarker proteoforms by hybrid LBA/LCMS

- Often, both active and inactive isoforms for protein biomarkers are included in hybrid LBA/LCMS assays. It is important to understand what is being measured and why since there is potential for diluting the effect by looking at all the proteoforms when only one is most important. Clearly define early on what your assay is measuring, not what is unknown.

- A biological matrix where only the endogenous protein biomarker was depleted while retaining all other biological background of the matrix can be used for hybrid LBA/LCMS as long as the surrogate matrix calibration is parallel and the endogenous levels allow for detectable concentrations changes.

- Aptamers for hybrid LBA/LCMS

- For poor affinity antibodies, consider flow-based antiprotein capture rather than bead-based methods to overcome poor antibody affinity.

- If antibodies used in IA are not available or are of poor specificity, aptamers can be explored as alternative affinity purification techniques. 
- The major benefit of aptamers is that all reagents are synthetic, resulting in less lot-to-lot variability and no issues with loss of antibody producing cell lines.

\section{- BAV for hybrid LBA/LCMS}

- Presently there is no regulatory guidance/guideline for hybrid LBA/LCMS. When validating a hybrid LBA/LCMS assay for protein biomarkers, a validation plan is needed listing experiments that are feasible to achieve the best answer to the question of the study and to have confidence in the assay performance, based on the needs of the results (i.e., primary or exploratory/secondary end point will drive decision for FFP or full validation). An understanding of the bias/risk that can occur during use of the assay is needed and should be addressed during validation.

= LCMS and hybrid LBA/LCMS have been included in the US FDA/Critical Path initiative to write a white paper for evidentiary standards for biomarker qualification.

\section{Acknowledgements}

The authors would like to acknowledge the US FDA, Europe EMA, UK MHRA, Austria AGES, France ANSM, Brazil ANVISA, Health Canada, Japan MHLW and Finland Fimea for supporting this workshop; S Gupta (Amgen), B DeSilva (Bristol-Myers Squibb), L Amaravadi (Sanofi), E Yang (GlaxoSmithKline), J Welink (EU EMA), A Song (Genentech), H Neubert (Pfizer), F Garofolo (Angelini Pharma) for chairing the workshop and/or the White Paper discussions; all the workshop attendees and members of the bioanalytical community who have sent comments and suggestions to complete this White Paper; and Future Science Group as a trusted partner.

\section{Financial \& competing interests disclosure}

The authors have no relevant affiliations or financial involvement with any organization or entity with a financial interest in or financial conflict with the subject matter or materials discussed in the manuscript. This includes employment, consultancies, honoraria, stock ownership or options, expert testimony, grants or patents received or pending, or royalties.

No writing assistance was utilized in the production of this manuscript.

\section{References}

1 Savoie N, Booth BP, Bradley T et al. 2008 White Paper: the 2nd Calibration and Validation Group Workshop on recent issues in good laboratory practice bioanalysis. Bioanalysis 1(1), 19-30 (2009).

2 Savoie N, Garofolo F, van Amsterdam P et al. 2009 White Paper on recent issues in regulated bioanalysis from the 3rd Calibration and Validation Group Workshop. Bioanalysis 2(1), 53-68 (2010).

3 Savoie N, Garofolo F, van Amsterdam P et al. 2010 White Paper on recent issues in regulated bioanalysis and global harmonization of bioanalytical guidance. Bioanalysis 2(12), 1945-1960 (2010).

4 Garofolo F, Rocci M, Dumont I et al. 2011 White Paper on recent issues in bioanalysis and regulatory findings from audits and inspections. Bioanalysis 3(18), 2081-2096 (2011).

5 DeSilva B, Garofolo F, Rocci M et al. 2012 White Paper on recent issues in bioanalysis and alignment of multiple guidelines. Bioanalysis 4(18), 2213-2226 (2012).

6 Stevenson L, Rocci M, Garofolo F et al. 2013 White Paper on recent issues in bioanalysis: "Hybrid" - the best of LBA \& LC/MS. Bioanalysis 5(23), 2903-2918 (2013).

7 Fluhler E, Hayes R, Garofolo F et al. 2014 White Paper on recent issues in bioanalysis: a full immersion in bioanalysis (Part 1 small molecules by LCMS). Bioanalysis 6(22), 3039-3049 (2014).

8 Dufield D, Neubert H, Garofolo F et al. 2014 White Paper on recent issues in bioanalysis: a full immersion in bioanalysis (Part 2 hybrid LBA/LCMS, ELN \& regulatory agencies' input). Bioanalysis 6(23), 3237-3249 (2014).

9 Stevenson L, Amaravadi L, Myler H et al. 2014 White Paper on recent issues in bioanalysis: a full immersion in bioanalysis (Part 3 - LBA and immunogenicity). Bioanalysis 6(24), 3355-3368 (2014).

10 Welink J, Fluhler E, Hughes N et al. 2015 White Paper on recent issues in bioanalysis: focus on new technologies and biomarkers (Part 1 - small molecules by LCMS). Bioanalysis 7(22), 2913-2925 (2015).

11 Ackermann B, Neubert H, Hughes N et al. 2015 White Paper on recent issues in bioanalysis: focus on new technologies and biomarkers (Part 2 - hybrid LBA/LCMS and input from regulatory agencies). Bioanalysis 7(23), 3019-3034 (2015).

12 Amaravadi L, Song A, Myler H et al. 2015 White Paper on recent issues in bioanalysis: focus on new technologies and biomarkers (Part 3 - LBA, biomarkers and immunogenicity). Bioanalysis 7(24), 3107-3124 (2015). 
13 Yang E, Welink J, Cape S et al. 2016 White Paper on recent issues in bioanalysis: focus on biomarker assay validation (BAV) (Part 1 - small molecules, peptides and small molecule biomarkers by LCMS). Bioanalysis 8(22), 2363-2378 (2016).

14 Song A, Lee A, Garofolo F et al. 2016 White Paper on recent issues in bioanalysis: focus on biomarker assay validation (BAV): (Part 2 - hybrid LBA/LCMS and input from regulatory agencies). Bioanalysis 8(23), 2457-2474 (2016).

15 Richards S, Amaravadi L, Pillutla R et al. 2016 White Paper on recent issues in bioanalysis: focus on biomarker assay validation (BAV): (Part 3 - LBA, biomarkers and immunogenicity). Bioanalysis 8(23), 2475-2496 (2016).

16 Jiang $\mathrm{H}, \mathrm{Xu}$ W, Titsch CA et al. Innovative use of LC-MS/MS for simultaneous quantitation of neutralizing antibody, residual drug, and human immunoglobulin G in immunogenicity assay development. Anal. Chem. 86(5), 2673-2680 (2014).

$17 \mathrm{Xu}$ W, Jiang H, Titsch CA et al. Development and characterization of a pre-treatment procedure to eliminate human monoclonal antibody therapeutic drug and matrix interference in cell-based functional neutralizing antibody assays. J. Immunol. Methods 416, 94-104 (2015).

18 Chen LZ, Roos D, Philip E. Development of immunocapture-LC/MS assay for simultaneous ADA isotyping and semiquantitation. J. Immunol. Res. (2016). http://dx.doi.org/10.1155/2016/7682472

19 Mehl JT, Sleczka BG, Ciccimaro EF et al. Quantification of in vivo site-specific Asp isomerization and Asn deamidation of $\mathrm{mAbs}$ in animal serum using IP-LC-MS. Bioanalysis 8(15), 1611-1622 (2016).

20 Ciccimaro E, Zhu Y, Ostanin D et al. Antibody drug-target engagement measurement in tissue using quantitative affinity extraction liquid chromatography-mass spectrometry: method development and qualification. Anal. Chem. 89(9), 5115-5123 (2017).

21 Palandra J, Quazi A, Fitz L et al. Quantitative measurements of GDF-8 using immunoaffinity LC-MS/MS. Proteomics Clin. Appl. 10(5), 597-604 (2016).

22 Telliez JB, Dowty ME, Wang LU et al. Discovery of a JAK3-selective inhibitor: functional differentiation of JAK3-selective inhibition over pan-JAK or JAK1-selective inhibition. ACS Chem. Biol. 11(12), 3442-3451 (2016).

23 Neubert H, Gale J, Muirhead D. Online high-flow peptide immunoaffinity enrichment and nanoflow LC-MS/MS: assay development for total salivary pepsin/pepsinogen. Clin. Chem. 56(9), 1413-1423 (2010).

24 Fan YY, Avery LB, Wang M et al. Tissue expression profile of human neonatal Fc receptor ( $\mathrm{FcRn})$ in $\mathrm{Tg} 32$ transgenic mice. MAbs 8(5), 848-853 (2016).

25 Whiteaker JR, Zhao L, Yan P et al. Peptide immunoaffinity enrichment and targeted mass spectrometry enables multiplex, quantitative pharmacodynamic studies of phospho-signaling. Mol. Cell Proteomics 14(8), 2261-2273 (2015).

26 Bateman RJ, Munsell LY, Morris JC et al. Human amyloid-bold beta synthesis and clearance rates as measured in cerebrospinal fluid in vivo. Nature Med. 12(7), 856-861 (2006).

27 Thomas SN, Yang AJ. Mass spectrometry analysis of lysine posttranslational modifications of tau protein from Alzheimer's disease brain. Methods Mol. Biol. 1523, 161-177 (2017).

28 Gupta V, Lassman ME, McAvoy T et al. An evaluation of an aptamer for use as an affinity reagent with MS: PCSK9 as an example protein. Bioanalysis 8(15), 1557-1564 (2016).

29 US Department of Health and Human Services, US FDA, Center for Drug Evaluation and Research, Center for Veterinary Medicine. Guidance for Industry, Bioanalytical Method Validation, MD, USA (2001).

https://www.fda.gov/downloads/Drugs/Guidance/ucm070107.pdf

30 European Medicines Agency. Committee for Medicinal Products for Human Use (CHMP). Guideline on Bioanalytical Method Validation. EMEA/CHMP/EWP/192217/2009, London, UK (2011).

https://www.fda.gov/downloads/Drugs/Guidance/ucm070107.pdf

31 MHLW. Guideline on Bioanalytical Method Validation in Pharmaceutical Development. Japan (2013). http://www.nihs.go.jp/drug/BMV/250913_BMV-GL_E.pdf

32 Duggan J, Ren B, Mao Y, Chen LZ, Philip. LCMS quantification of protein drugs: validating protein LC-MS methods with predigestion immunocapture. Bioanalysis 8(18), 1951-1964 (2016).

33 Gupta S, Richards S, Amaravadi L et al. 2017 White Paper on recent issues in bioanalysis: a global perspective on immunogenicity guidelines \& biomarker assays performance (Part 3 - LBA: immunogenicity, biomarkers and PK assays). Bioanalysis 9(24), (2017).

34 Hougton R, Gouty D, Allinson J et al. Recommendations on biomarker bioanalytical method validation by GCC Bioanalysis 4(20), 2439-2446 (2012).

35 Piccoli S, Sauer JM, Amur S et al. Scientific and regulatory considerations for the analytical validation of assays used in the qualification of biomarkers in biological matrices.

https://healthpolicy.duke.edu/sites/default/files/atoms/files/white_paper_6_14_17_0.pdf

36 Health Canada. Notice: proposed policy on bioequivalence standards for highly variable drug products (2016). https:/www.canada.ca/en/health-canada/services/drugs-health-products/drug-products/announcements/notice-policybioequivalence-standards-highly-variable-drug-products.html

37 Health Canada. Notice: submission filing requirements - good manufacturing practices (GMP)/drug establishment licenses 
(DEL) (2017). https://www.canada.ca/en/health-canada/services/drugs-health-products/drug-products/applications-submissions/ guidance-documents/notice-submission-filing-requirements-good-manufacturing-practices-establishment-licences.html

38 Health Canada. Bureau of Pharmaceutical Sciences Therapeutic Products Directorate. Notice: clarification of bioanalytical method validation procedures (2015). https://www.canada.ca/en/health-canada/services/drugs-health-products/drug-products/ announcements/notice-clarification-bioanalytical-method-validation-procedures.html

39 Health Canada. Bureau of Pharmaceutical Sciences Therapeutic Products Directorate. Addendum to Notice: Clarification of Bioanalytical Method Validation Procedures (2016). https://www.canada.ca/en/health-canada/services/drugs-health-products/ drug-products/announcements/notice-clarification-bioanalytical-method-validation-procedures.html\#a1

40 Welink J, Yang E, Hughes N et al. 2017 White Paper on recent issues in bioanalysis: aren't BMV/guidance/guidelines 'Scientific'? (Part 1 - LCMS: small molecules, peptides and small molecule biomarkers). Bioanalysis 9(22), 1807-1825 (2017).

41 ICH Final Endorsed Concept Paper M10: Bioanalytical Method Validation (2016). http://www.ich.org/fileadmin/Public_Web_ Site/ICH_Products/Guidelines/Multidisciplinary/M10/ICH_M10_Concept_paper_final_7Oct2016.pdf

42 ICH Final Endorsed Business Plan M10: Bioanalytical Method Validation (2016). http://www.ich.org/fileadmin/Public_Web_ Site/ICH_Products/Guidelines/Multidisciplinary/M10/ICH_M10_Bussiness_plan_final_7Oct2016.pdf

43 ICH M10 EWG Work Plan (2017). http://www.ich.org/fileadmin/Public_Web_Site/ICH_Products/Guidelines/Multidisciplinary/ M10/M10_EWG_Work_Plan_10Mar2017.pdf

\section{Affiliations}

${ }^{1}$ Pfizer, Andover, MA, USA

${ }^{2}$ Genentech, South San Francisco, CA, USA

${ }^{3}$ Merck, Kenilworth, NJ, USA

${ }^{4}$ Vertex Pharmaceuticals, Boston, MA, USA

5JXD Bioanalytics, Southbury, CT, USA (previously Boehringer-

Ingelheim, Ridgefield, CT, USA)

${ }^{6}$ Merck, West Point, PA, USA

${ }^{7}$ GlaxoSmithKline, King of Prussia, PA, USA

${ }^{8}$ US FDA, Silver Spring, MD, USA

${ }^{9}$ Austria AGES, Wien, Austria

${ }^{10} \mathrm{Health}$ Canada, Ottawa, ON, Canada

${ }^{11}$ Bristol-Myers Squibb, Princeton, NJ, USA

${ }^{12}$ UK MHRA-NIBSC, London, UK

${ }^{13} \mathrm{AbbVie}$ Stemcentrx, South San Francisco, CA, USA

${ }^{14}$ Caprion BioSciences, Montreal, QC, Canada

${ }^{15}$ Angelini Pharma, Rome, Italy

${ }^{16} J a p a n$ MHLW-NIHS, Tokyo, Japan

\author{
${ }^{17}$ Finland Fimea, Helsinki, Finland \\ ${ }^{18}$ France ANSM, Saint Denis, France \\ ${ }^{19}$ Regulus, San Diego, CA, USA \\ ${ }^{20}$ Brazil Anvisa, Brasilia, Brazil \\ ${ }^{21}$ Nippon Kayaku, Tokyo, Japan \\ ${ }^{22}$ Takeda Pharmaceuticals, Cambridge, MA, USA \\ ${ }^{23}$ Pfizer, Groton, CT, USA \\ ${ }^{24}$ CFABS, Montreal, QC, Canada \\ ${ }^{25}$ UK MHRA, London, UK \\ ${ }^{26}$ EMA, London, UK \\ ${ }^{27}$ AstraZeneca, Cambridge, UK \\ ${ }^{28}$ Celgene, Summit, NJ, USA \\ *Author for correspondence: fabiogarofolo@cfabs.org
}

